\title{
A Clinical Predictive Model for Catheter Related Bloodstream Infections from the Electronic Medical Record
}

\author{
Gonzalo M.L. Bearman ", Michael I. Oppenheim, Eneida A. Mendonca, Nathaniel Hupert, \\ Maryam Behta, Paul J. Christos and Lewis M. Drusin
}

VCU Medical Center, Richmond, Box 980019, VA 23298-0019, USA

\begin{abstract}
Background and Methods: Previous studies identifying risk factors for catheter related blood stream infections (BSI) have relied on labor-intensive paper chart review. The electronic medical record (EMR) can provide real-time data for profiling patients at risk for BSI. Our study's aim was to use our hospital EMR to identify risk factors and develop a clinical predictive model for catheter related BSI from June 2001-June 2002. Using a modified CDC definition for BSI, we retrospectively extracted a number of variables from the EMR data repository including demographics, medications, nutritional status, presence of diabetes (DM), skin/wound status, use of a mechanical ventilator, and presence of a urinary catheter or central/arterial/PICC catheter. We developed a Cox Proportional Hazards model using half the cohort (14,977 controls and 123 BSI cases), and validated the model using the other half. Final variables in the multivariate model were chosen by clinical relevance, significance on univariate analysis and by step-wise automated selection.

Results: The final model contained: use of antibiotics (HR 4.6), central venous catheter for 0-6 days (HR 1.8), central venous catheter for greater or equal to 7 days (HR 1.3), tunneled central venous port (HR 9.3) and DM (HR 2.1). Variables from multivariate analysis were used for the predictive index; points were derived from the respective HR of the Cox model. A cutoff value that maximized sensitivity (75\%) and specificity ( $89 \%)$ had a positive predictive value (PPV) of .05 . This model was validated on the second half of the cohort (122 BSI, 14,977 'non-cases') with resultant sensitivity of $69 \%$, specificity of $88 \%$ and PPV of 0.05 . Comparison of the two models revealed no significant differences in the sensitivity, specificity and PPV.

Conclusion: We successfully used a hospital-based EMR in developing a Cox Proportional Hazards model for catheter related BSI. This model was used to derive a predictive index. At a cutoff point providing a sensitivity of $75 \%$ and specificity of $89 \%$ for finding patients who develop catheter related BSI, the index had a PPV 0.05 . When applied to a predictive index, real time data gathered from the EMR may assist in identifying patients at risk for a catheter related BSI and may be further utilized as part of a protocol driven, preventive strategy for catheter related BSI risk reduction.
\end{abstract}

Keywords: Electronic medical record, catheter related -bloodstream infection, clinical predictive modeling.

\section{BACKGROUND}

Catheter related bloodstream infections are a leading cause of death in the United States and, despite advances in medical treatments, remain a serious medical problem. A recent meta-analysis reviewing greater than 50 years of published reports concluded that catheter related bloodstream infections in critically ill adult patients was associated with a significantly increased mortality, even in subgroup analyses that were matched for severity of illness [1]. In both developed and developing countries, catheter related bloodstream infections were associated with increased duration of mechanical ventilation, prolonged ICU and hospital stay, and excesses in cost $[2,3]$.

Risk factors for catheter related bloodstream infections (BSI) include the introduction of bacteria into the circulation through invasive central venous and arterial catheters, tunneled central catheters, and peripherally inserted central lines and intravenous lines. Additionally, multiple factors

*Address correspondence to this author at the VCU Medical Center, Richmond, Box 980019, VA 23298-0019, USA; Tel: 804.828.2121; Fax: 804.828.2125; E-mail: gbearman@mcvh-vcu.edu can lead to primary infection with secondary bacteremia including head injury, sedation, malnutrition, immunosuppression, mechanical ventilation and surgery.

Many prior epidemiologic studies of catheter related BSI have been retrospective and case-control in design. The electronic medical record (EMR) affords the opportunity to identify risk factors in real-time. The application of concurrent risk identification may prove to be a crucial step for risk factor reduction interventions in hospitalized patients.

We sought to utilize data from our hospital EMR to develop a clinical predictive model for catheter related BSI. We retrospectively analyzed a one-year cohort of hospitalized patients in a tertiary care medical center in order to develop a Cox-Proportional Hazards model for catheter related BSI. This multivariate model was derived from half of the hospitalized cohort. From the Cox model, a predictive index was developed, with the final 'score' chosen to maximize sensitivity, specificity and positive predictive value. The predictive index was later validated on the second half of the cohort. 


\section{METHODS}

The retrospective study was performed at an 850 bed tertiary care medical center located in New York City. The medical center provides multi-specialty services including medicine, surgery, cardiology, cardiothoracic surgery, obstetrics and gynecology, pediatrics and bone and solid organ transplantation. The medical center utilizes a commercial inpatient prescriber order entry and clinical documentation system (Sunrise Critical Care v1.3, Eclipsys Corporation, Boca Raton, Fl). One hundred percent of nursing and ancillary service documentation is done electronically. Additionally, physicians on a large majority of the non-pediatric inpatient services are entering $100 \%$ of their orders into the system (the excluded services are Obstetrics \& gynecology, burn, and cardiothoracic surgery). All data entered into the clinical information system is copied into a clinical data repository and is available for retrospective review and queries.

The time frame for analysis was June 1, 2001 to June 1, 2002. A case of primary, catheter related BSI was defined using a modified Centers for Disease Control Criteria (CDC), as follows. A 1st episode of catheter related BSI was considered in all patients with newly positive blood cultures $\geq 48$ hours post admission. Patients with positive blood cultures within the first 48 hours of admission were excluded along with patient with the same organism isolated from blood cultures pre and post 48 hours of hospitalization. All cases with coagulase negative staphylococci and other skin commensals such a Proprionobacterium acnes and Corynebacteria species were also excluded from analysis as it is difficult to distinguish between clinically relevant isolates versus contamination based solely on EMR data. We extracted a number of clinical variables from the EMR data repository including demographics, medications, nutritional status, presence of diabetes (DM), skin/wound status, use and duration of mechanical ventilation, urinary catheterization, central, arterial, and peripherally inserted central venous catheters. Medications included receipt and duration of use of antibiotics, sedatives, $\mathrm{H} 2$ blockers, proton pump inhibitors, immunosuppressive medications and diabetic medications. Nutritional status was dichotomized as presence/absence of malnutrition as documented by the Hospital's Dietary Service patient nutritional assessment. Skin/wound status was extracted from the decubitus ulcer wound category (ordinal data, classes I-V) documented in the nurses daily report. We developed a Cox Proportional Hazards model with half the cohort (14,977 controls and 123 BSI cases). All data was collected up until the day of the 1st positive blood culture. Final variables were chosen by clinical relevance, significance on univariate analysis and by step-wise automated selection to include confounders. All data was extracted from the companion clinical data respository of the hospital's EMR. The final model was then validated using the second half of the cohort. A prediction rule was built using the results of the multivariate analysis which applies a weight to each of the clinical variables to determine a risk score. The prediction rule was tested using the other half of our patient cohort. The choice of Cox Proportional Hazards model for analytic technique was due to the availability of person-time data from the database.
Also, this model is appropriate for unequal observation periods.

\section{RESULTS}

In the total cohort, 246 distinct NBSI cases were identified. There were 29,955 non-cases. From this population, the test cohort was defined and contained 125 NBSI cases and 14,972 non-cases. The test model contained: use of antibiotics (HR 4.6), consecutive central venous catheter for 0-6 days (HR 1.8), consecutive central venous catheter for greater or equal to 7 days (HR 1.3) tunneled central venous port (HR 9.3) and DM (HR 2.1, Table 1). Variables from multivariate analysis were used for the predictive index; points were derived from the respective HR of the Cox model. The assigned points were as follows; receipt of antimicrobials prior to catheter related BSI event 5 pts, presence of CVC for 1-6 days 2 pts, presence of CVC for greater than or equal to 7 days $1 \mathrm{pt}$, diabetes mellitus $2 \mathrm{pts}$ and presence of a tunneled CVC for greater than 7 days, 9 pts. A cutoff value that maximized sensitivity $(75 \%)$ and specificity $(89 \%)$ had a positive predictive value (PPV) of .05 (Table 2). This model was validated on the second half of the cohort (121 BSI, 14,973 'non-cases') with resultant sensitivity of $69 \%$, specificity of $88 \%$ and PPV of 0.046 (Table 3). Comparison of the two models revealed no significant difference in sensitivity (P 0.3296), specificity (P $0.5080)$ and positive predictive value (P 0.4220, Table 4).

Table 1. Multivariate Model from Test Cohort

\begin{tabular}{|c|c|c|}
\hline Variable & Hazard Ratio & P Value \\
\hline \hline Receipt of Antimicrobial & 4.6 & $<0.0001$ \\
\hline CVC for 1-6 days & 1.8 & 0.0178 \\
\hline CVC for $>7$ days & 1.3 & 0.3297 \\
\hline Tunneled CVC for $>7$ days & 9.3 & $<0.0001$ \\
\hline Diabetes mellitus & 2.1 & 0.0002 \\
\hline
\end{tabular}

All variables were chosen by clinical relevance, significance on univariate analysis, and by SAS step-wise selection.

CVC use greater than or equal to 7 days was retained in the model due to pathophysiologic plausibility.

\section{DISCUSSION}

Catheter related BSIs are important causes of morbidity and mortality. On a national level, catheter related bacteremias are the 10th leading cause of death in the United States [4]. Additionally, the age adjusted death rate has increased by $78 \%$ over the last 20 years [5]. Although the exact incidence of catheter related blood stream infections is unknown, it is believed that approximately 250,000 cases occur annually in the United States [6, 7]. Data from the Surveillance and Control of Pathogens of Epidemiologic Importance [SCOPE]), which analyzed 24,170 cases of catheter related blood stream infections from 1995 to 2002, reported an incidence of 60 cases per 10,000 hospital admissions [7]. Within the hospital setting, the incidence of catheter related BSIs appears heterogeneous across different care environments. Recent publications suggest that the incidence of catheter related bacteremia ranges from $1 \%$ in 
Table 2. Predictive Model from Test Cohort

\begin{tabular}{|c|c|c|c|c|c|c|}
\hline Score & $\mathbf{N}$ with event & N without Event & Cutoff (if $</=$ Value, No Risk) & Positive Predictive Value & Sensitivity & Specificity \\
\hline 0 & 3 & 8637 & 0 & 0.019 & 0.976 & 0.577 \\
\hline 2 & 8 & 1425 & 2 & 0.022 & 0.911 & 0.672 \\
\hline 4 & 2 & 85 & 4 & 0.022 & 0.895 & 0.678 \\
\hline 5 & 19 & 3103 & 5 & 0.051 & 0.750 & 0.885 \\
\hline 7 & 39 & 1300 & 7 & 0.113 & 0.435 & 0.972 \\
\hline 9 & 44 & 398 & 9 & 0.294 & 0.081 & 0.998 \\
\hline 13 & 0 & 3 & 13 & 0.323 & 0.081 & 0.999 \\
\hline 18 & 8 & 13 & 18 & 0.200 & 0.016 & 0.999 \\
\hline
\end{tabular}

Variables from the multivariate analysis were used for the predictive index. Points were derived from the respective hazard ratio of the Cox Proportional Hazards Model:

- Receipt of antimicrobials prior to NBSI event 5 pts.

- $\quad$ Presence of CVC for 1-6 days 2 pts.

- $\quad$ Presence of CVC for greater than or equal to 7 days $1 \mathrm{pt}$.

- $\quad$ Diabetes mellitus 2 pts.

- $\quad$ Tunneled CVC fro greater than 7 days- 9pts.

Table 3. Validation Model

\begin{tabular}{|c|c|c|c|c|c|c|}
\hline Score & N with Event & N without Event & Cutoff (if $</=$ Value, No Risk) & Positive Predictive Value & Sensitivity & Specificity \\
\hline 0 & 6 & 8565 & 0 & 0.018 & 0.950 & 0.572 \\
\hline 1 & 5 & 156 & 1 & 0.017 & 0.909 & 0.582 \\
\hline 2 & 2 & 1297 & 2 & 0.021 & 0.893 & 0.669 \\
\hline 3 & 3 & 23 & 3 & 0.021 & 0.868 & 0.670 \\
\hline 4 & 0 & 74 & 4 & 0.021 & 0.868 & 0.675 \\
\hline 5 & 21 & 3108 & 5 & 0.046 & 0.694 & 0.883 \\
\hline 6 & 10 & 180 & 6 & 0.045 & 0.612 & 0.895 \\
\hline 7 & 34 & 1153 & 7 & 0.086 & 0.331 & 0.972 \\
\hline 8 & 16 & 178 & 8 & 0.088 & 0.198 & 0.983 \\
\hline 9 & 18 & 207 & 9 & 0.125 & 0.050 & 0.997 \\
\hline 10 & 0 & 9 & 10 & 0.154 & 0.050 & 0.998 \\
\hline 12 & 0 & 4 & 12 & 0.171 & 0.050 & 0.998 \\
\hline 15 & 4 & 19 & 15 & 0.167 & 0.017 & 0.999 \\
\hline
\end{tabular}

Variables from the multivariate analysis were used for the predictive index. Points were derived from the respective hazard ratio of the Cox Proportional Hazards Model:

- Receipt of antimicrobials prior to NBSI event 5 pts.

- $\quad$ Presence of CVC for 1-6 days 2 pts.

- $\quad$ Presence of CVC for greater than or equal to 7 days $1 \mathrm{pt}$.

- $\quad$ Diabetes mellitus 2 pts.

- $\quad$ Tunneled CVC fro greater than 7 days- 9pts.

Table 4. Internal Validation of Predictive Model- Positive Predictive Value, Sensitivity and Specificity

\begin{tabular}{|c|c|c|c|c|}
\hline Parameter & Predictive Model & Validation Model & Test Statistic & P Value \\
\hline \hline Positive Predictive Value & 0.051 & 0.046 & 0.6425 & 0.4220 \\
\hline Sensitivity & 0.750 & 0.694 & 0.9504 & 0.3296 \\
\hline Specificity & 0.885 & 0.883 & 0.4382 & 0.5080 \\
\hline
\end{tabular}

intensive care unit populations to as high as $36 \%$ in bone marrow transplant recipients [8, 9]. In a recent study analyzing catheter related BSIs caused by antibiotic resistant gram negative bacteria in critically ill patients, the incidence of bacteremia was 11 cases per 1000 ICU admissions [10]. Lastly, data from a 12 year retrospective review of catheter 
related BSIs in a 900 bed, tertiary care hospital, documented a linear increase of crude infection rates from 6.7 to 188.4 per 1000 discharges [1]. Thus, reports on incidence of catheter related bloodstream infection vary significantly likely reflecting differences in individual risk based on institution, population type, comorbid illnesses, length of stay and hospital location.

Both the prevention and management of BSIs will continue to challenge physicians as reports of pathogen resistance increase. However, the EMR may be a useful adjunct in BSI prevention. Traditional infection control programs involve surveillance and feedback of outcome measures, such as bloodstream infection and ventilatorassociated pneumonia rates. Outcome measures are, however, based on uncommon events that take longer to observe and also may not directly relate to individual or group performance as they are frequently affected by factors related to the patient and the healthcare system. In contrast, process measures are attractive for several reasons. They provide operational and measurable representation of performance, relate to individual and/or group performance, and are easier to measure than outcomes. By potentially increasing overall accountability, they create opportunities to monitor and improve performance. Provided a process measure and outcome are linked, improvements in process measure compliance may theoretically improve outcomes. Personalized automated reminders or flags that are designed to improve processes of care have been shown to lead to better patient outcomes, but they must be tailored to both the acuity of the process and frequency of false positives to minimize "alert fatigue" [11].

Berenholtz et al. studied the effect of a multifaceted systems intervention on catheter related bloodstream infections in the intensive care unit [12]. The strategy included a quality improvement team that implemented five interventions. These were [1] staff education [2], the creation of a catheter insertion cart to minimize the steps of catheter insertion and (3) the standardized questioning of daily providers whether catheters could be removed. Most importantly [4], a checklist to ensure adherence to evidencebased guidelines for preventing CR-BSIs was implemented along with [5] the empowerment of nurses to stop the catheter insertion procedure if a violation of the guidelines was observed. Evidence based process-of-care measures for catheter insertion included a nurse's checklist for observed hand hygiene, patient skin antisepsis, proper use of sterile gloves, gown and drape. Additionally, the checklist included confirmation that all personnel complied with infection control precautions. Following the intervention, the CR-BSI rate in the study ICU decreased from 11.3/1,000 catheter days in the first quarter of 1998 to $0 / 1,000$ catheter days in the fourth quarter of 2002. The CR-BSI rate in the control ICU was 5.7/1,000 catheter days in the first quarter of 1998 and 1.6/1,000 catheter days in the fourth quarter of $2002(\mathrm{p}=$ .56). As per the estimates of Berenholtz et al., the initiative may have prevented 43 CR-BSIs, eight deaths, and $\$ 1,945,922$ in additional costs per year in the study ICU. Pronovost et al., using the same risk reduction protocol, achieved similar results across 103 Michigan ICUs [13].

Wall et al. studied the impact of process measure interventions in the reduction of catheter related bloodstream infections in the intensive care unit [14]. An interdisciplinary team developed a standardized, nursing checklist for CVC insertion. Infection control practitioners scanned the completed checklists into a computerized database, thereby generating real time measurements for the process of CVC insertion. These infection control process measures allowed the ICU team to directly monitor adherence to evidencebased guidelines. After two years, the investigators reported a persistent and historically low catheter related bloodstream infection rate, demonstrating that multifaceted programs ensuring maximal adherence with evidence-based infection control guidelines are effective in reducing the incidence of catheter related bloodstream infections in the intensive care setting [14].

The EMR can be further utilized as part of a protocol driven, preventive strategy for catheter related BSI risk reduction. As part of the risk reduction process of care interventions, the daily assessment of the necessity of ongoing catherization can be counterbalanced with a quantifiable, evidence based risk of a catheter related BSI. For instance, as part of the daily rounds, data from the EMR could be used to generate a hazard analysis and corresponding predictive index for the development of a catheter related bloodstream infection in a given patient. Based on the predictive index and its sensitivity, specificity and positive predictive value, decisions regarding central venous catheter discontinuation can become more evidencebased, ultimately leading to more standardized and uniformly implemented risk reduction strategies for the prevention of catheter related bloodstream infections. Recognizing that central venous catheters are the primary risk factor for catheter related BSIs, risk reduction may be attempted with multi-level interventions, including evidence based, best practices insertion and maintenance protocols, and daily review of ongoing catheterization. If applied in real time, clinical data automatically generated from the EMR may be used for predictive modeling of catheter related BSIs, thus providing medical teams with a patient specific BSI point score, hazard ratio and a predictive value to assist in decision management for discontinuation of catheterization.

Our study has several limitations. All of the data was collected retrospectively. Data on central line placement, duration and anatomic location were all subject to misclassification bias by hospital personnel at the time of data entry into the EMR. The predictive index was developed on a retrospective cohort and has not been prospectively validated. Nevertheless, both the predictive and validation cohorts were large in size. Thus, the power of the study was large, minimizing the risk of a type II error. In addition, we used a modified CDC definition for catheter related BSI which excluded all potential cases with coagulase negative staphylococcal isolates in the blood given the inability to accurately classify these isolates as common skin contaminants versus clinically relevant isolates based solely by automated EMR data. Recognizing that coagulase negative staphylococci are important catheter related bloodstream infection pathogens, the model is thus valid only for catheter related BSIs caused by other pathogens such $S$. aureus, gram negative pathogens, enterococci and candida species. Future implementation of similar clinical predictive models would require inclusion of coagulase 
negative staphylococci for greater application. Lastly, all of the data was collected from a single large, tertiary care medical center, potentially impacting the ability to generalize the model to other centers.

We successfully used a hospital-based EMR to retrospectively develop a Cox Proportional Hazards model for catheter related BSI. This model was used to derive a predictive index. At a cutoff point providing a sensitivity of $75 \%$ and specificity of $89 \%$ for finding patients who develop catheter related BSI, the index had a PPV 0.05. This predictive index must be prospectively validated. In our cohort, PPV was low because of the low incidence of catheter related BSIs. PPV would be higher in situations with a higher incidence of catheter related BSI and thus may be of greater use in settings with elevated infection rates. EMR based predictive index for catheter related bloodstream infections may assist healthcare providers in an evidence based risk reduction interventions for limiting catheter related bloodstream infections.

\section{REFERENCES}

[1] Siempos II, Kopterides P, Tsangaris I, Dimopoulou I, Armaganidis AE. Impact of catheter-related bloodstream infections on the mortality of critically ill patients: a meta analysis. Crit Care Med 2009; 37: 2283-9.

[2] Blot SI, Depuydt P, Annemans L, et al. Clinical and economic outcomes in critically ill patients with nosocomial catheter-related bloodstream infections. Clin Infect Dis 2005; 41: 1591-8.

[3] Rosenthal V, Guzman S, Migone O, Crnich C. The attributable cost, length of hospital stay, and mortality of central line-associated bloodstream infection in intensive care departments in Argentina: a prospective, matched analysis. Am J Infect Control 2003; 31: 47580.

[4] Freid V, Prager K, MacKay A, Xia H. Health, United States, 2003. Hyattsville, Maryland: National Center for Health Statistics; 2003.

[5] National Nosocomial Infections Surveillance (NNIS) System Report, Data Summary from January 1992-April 2000, Issued June 2000. Am J Infect Control 2000; 28: 429-48.

[6] Pittet D, Li N, Woolson R. Microbiological factors influencing the outcome of nosocomial bloodstream infections: a 6-year validated, populations-based model. Clin Infect Dis 1997; 24: 1068-78.

[7] Wisplinghoff $H$, Bischoff $T$, Tallent $S$, Seifert $H$, Wenzel $R$, Edmond M. Nosocomial bloodstream infections in US hospitals: analysis of 24,179 cases from a prospective nationwide surveillance study. Clin Infect Dis 2004; 39: 309-17.

[8] Warren D, Zack J, Elward A, Cox M, Fraser V. Nosocomial primary bloodstream infections in intensive care unit patients in a nonteaching community medical center: a 21 -month prospective study. Clin Infect Dis 2001; 33: 1329-35.

[9] Collin B, Leather H, Wingard J, Ramphal R. Evolution, incidence, and susceptibility of bacterial bloodstream isolated from 519 bone marrow transplant patients. Clin Infect Dis 2001; 33: 947-53.

[10] Blot S, Vandewoude K, De Bacquer D, Colardyn F. Nosocomial bacteremia caused by antibiotic-resistant gram-negative bacteria in critically ill patients: clinical outcome and length of hospitalization. Clin Infect Dis 2002; 34: 1600-6.

[11] Luna D, Otero V, Canosa D, Montenegro S, Otero P, de Quirós FG. Analysis and redesign of a knowledge database for a drug-drug interactions alert system. Studn Health Technol Inform 2007; 12(Pt 2): 885-9.

[12] Berenholtz SM, Pronovost PJ, Lipsett PA, et al. Eliminating catheter-related bloodstream infections in the intensive care unit. Crit Care Med 2004; 32: 2014-20.

[13] Pronovost $\mathrm{P}$, Needham D, Berenholtz $\mathrm{S}$, et al. An intervention to decrease catheter-related bloodstream infections in the ICU. N Engl J Med 2006; 355: 2725-32.

[14] Wall RJ, Ely EW, Elasy TA, et al. Using real time process measurements to reduce catheter related bloodstream infections in the intensive care unit. Qual Saf Health Care 2005; 14: 295-302. 